\title{
ARTÍGULO ORIGINAL: Indicadores de Calidad en la Unidad de Hemodiálisis del Hospital Rafael Ángel Calderón Guardia
}

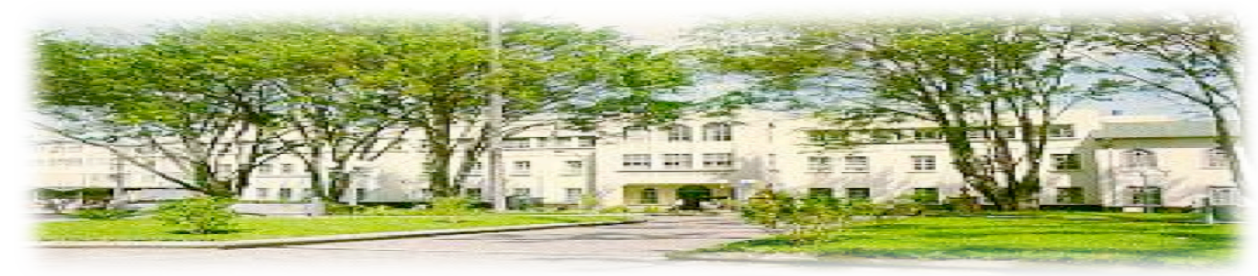

Haspital San quan de Dias. San Jasé. Casta Rica. Fundada en 1845

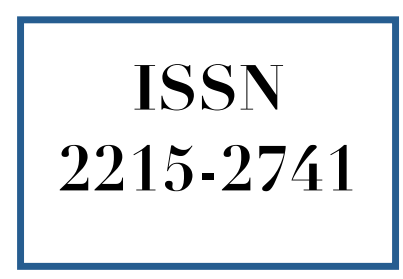

$\begin{array}{ll}\text { Recibido: } & 07 / 05 / 2013 \\ \text { Aceptado: } & 17 / 07 / 2013\end{array}$

\author{
Mario Espinach Roel ${ }^{1}$ \\ Marta Avellán Boza ${ }^{2}$
}

${ }^{\mathbf{1}}$ Médico Especialista en Medicina Interna y Nefrología UCR-CENDEISSS. Hospital Rafael Ángel Calderón Guardia. San José. Costa Rica. Correo electrónico: mer1402@gmail.com

${ }^{2}$ Médica Especialista en Medicina Interna y Residente de Nefrología. PPEM UCR-CENDEISSS. Hospital Rafael Ángel Calderón Guardia. San José. Costa Rica. Correo electrónico: martavellan@gmail.com

\section{RESUMEN}

Introducción: En los últimos años, la comunidad científica ha puesto de manifiesto el interés por estandarizar el cuidado de los pacientes en diálisis. Este interés se pone de manifiesto en la publicación de actualizaciones y guías en distintos países, que seleccionan criterios de calidad, cuyo grado de cumplimiento debe ser medido. A dichas medidas se les llama indicadores. En el Hospital Calderón Guardia (HCG) hay alrededor de 30 pacientes en programa de hemodiálisis crónica, los mismos no cumplen con las horas de terapia dialítica recomendadas por las guías internacionales, por lo que se sospecha que tampoco alcanzan las metas, en lo que a los indicadores de calidad se refiere. Por esta razón, el objetivo del presente estudio es presentar los primeros resultados sobre indicadores de calidad obtenidos en la Unidad de Hemodiálisis del HCG, a partir de los cuales se detecte los puntos débiles y se proponga soluciones para mejorar los resultados a futuro. Materiales y métodos: Estudio Observacional, retrospectivo, de los indicadores obtenidos en el HCG en el año 2012 y primer trimestre del 2013, cuyos datos fueron registrados durante este periodo en una base de datos de Excel. Resultados: De los indicadores de calidad analizados en este estudio, las metas se alcanzaron únicamente en lo que se refiere a las infecciones virales y los niveles de colesterol LDL (menor a $100 \mathrm{mg} / \mathrm{dl}$ ), para los demás indicadores, se está lejos de los objetivos recomendados por las guías internacionales. Discusión: Este estudio evidencia el mal cumplimiento de los criterios de calidad de la hemodiálisis, evidenciado por la dosificación de diálisis subóptima y la persistencia de reactantes de fase aguda estimulados (PCR alta y albúmina baja), hecho que incide directamente en los demás indicadores, como los los niveles de 
hemoglobina, que es uno de los cuales está más lejos de alcanzar la meta, a pesar de dosis altas de eritropoyetina. En cuanto al metabolismo mineral óseo, cabe destacar que la seguridad social no cuenta por el momento con quelantes de fósforo libres de calcio y de aluminio, lo cual se ve reflejado directamente en los altos niveles de fósforo y del producto calcio*fósforo. Los indicadores de calidad que sí alcanzaron los objetivos incluyen los que tienen que ver con infecciones víricas y los niveles de LDL colesterol. El primero es consecuencia directa del trabajo conjunto con el servicio de Infectología y el segundo probablemente en relación con un estado de malnutrición de los pacientes en hemodiálisis, que se ve reflejado también en los bajos niveles de albúmina. Conclusión: Los malos resultados demostrados en este estudio son interpretados como un avance en el conocimiento de la hemodiálisis, pero que evidencian el largo trabajo pendiente para mejorar los índices de calidad y permite desarrollar estrategias para mejorar cada indicador, según los recursos que se tienen en nuestro medio

\section{PALABRAS CLAVE}

Hemodiálisis. Indicadores de Calidad.

\section{ABSTRACT}

Introduction: In the past few years, the international scientific community had issue clear interest tending to standardize the care of dialysis patients. This interest is consolidated with the release of updates and guides in different countries. This guides selected quality criteria, whose degree of compliance should be measured, and they are called indicators. At the HCG there are about 30 patients in chronic hemodialysis program. They do not meet the hours of dialytic therapy recommended by international guidelines so the study group suspected that they do not reached the goals in regard to quality indicators. For this reason, the objective of the present study is to present the first results on quality indicators, obtained at the unit of hemodialysis of the HCG, from which weak points are detected and aim to propose solutions to improve outcomes in the future. Materials and methods: This a an observational retrospective study, of the indicators obtained at the HCG in 2012 and the first trimester of 2013, whose data were recorded during this period, as Excel database. The goals in the indicators, analyzed in this study that were achieved, were only in those that related to viral infections and cholesterol LDL less than 100 $\mathrm{mg} / \mathrm{dl}$, the other indicators are far from the objectives recommended by international guidelines. Discussion: This study demonstrates the poor quality of hemodialysis, which is clear in the insufficient dialysis dosis and by the persistence of stimulated acute phase reactants (high PCR, low albumin). This impacts directly to the other indicators, including the levels of hemoglobin, which is one of the indicators that is far away from the goal, despite high doses of erythropoietin. It should be noted that the social security in Costa Rica does not have, at least at the present tim, calcium free or aluminum free, phosphorus binders which is directly reflected in high levels of phosphorus and calcium*phosphorus product match. Indicators of quality that reach the goals includes the ones having to do with viral infections and the levels of LDL cholesterol, the first one is a direct result of a joint work with Infectology and the latter probably was related to a malnutrition state in hemodialysis patients, also reflected in the low levels of albumin. Conclusion: The bad results shown in this study are interpreted as an advance in the knowledge of a hemodialysis program, but they demonstrate the long work that is pending to do, tending to help us to develop strategies with the aim of improving each indicator, according to the resources that are in our midst.

\section{KEY WORDS}

Hemodialysis. Clinical-performance measures.

\section{INTRODUCCIÓN}

\section{Introducción}

La mortalidad en los pacientes con enfermedad renal terminal en Estados Unidos ha disminuido en los últimos dos años a pesar del incremento en la prevalencia de diabetes y del envejecimiento de la población ${ }^{(1)}$. Esta disminución ha sido del $22 \%$ durante los primeros 2 años y alrededor de $15 \%$ en los tres años siguientes ${ }^{(1)}$. A pesar de esta mejoría en la sobrevivencia temprana, la mortalidad persiste alta en los pacientes que son sometidos a terapia de hemodiálisis, de modo que fallecen aproximadamente 212 por cada 
1000 pacientes anualmente ${ }^{(2)}$. Se ha visto que mejorar la adecuación de diálisis y controlar la anemia está asociado con mejoría tanto de la sobrevida como de la calidad de vida de los pacientes con insuficiencia renal crónica ${ }^{(2,3)}$.

En los últimos diez años, la publicación de las Guías de la Kidney Disease Outcomes Quality Initiative (KDOQI) ${ }^{(4)}$ pone por primera vez de manifiesto el interés internacional por estandarizar el cuidado de los pacientes en diálisis. Este interés se ve consolidado con la publicación de posteriores actualizaciones y guías en distintos países, incluidas las guías desarrolladas por la Sociedad Española de Nefrología ${ }^{(5)}$. Estas guías han permitido que se estandaricen los distintos aspectos del tratamiento renal sustitutivo con hemodiálisis. El éxito de una guía sobre el cuidado de los pacientes depende en gran parte de su proceso de medida y de la difusión de los resultados.

Las guías seleccionan los criterios de calidad cuyo grado de cumplimento debe ser medida y a éstos se le llama indicadores. Una guía tiene que ser explícita, comprensible, cuantificable y aceptada por la comunidad científica. El indicador consiste en definir cómo se va a medir, es decir, es una medida cuantitativa para evaluar un criterio. Los indicadores se clasifican en informativos y de cumplimiento y se distribuyen por áreas que contemplan distintos aspectos de la hemodiálisis. Entre los informativos se incluyen: datos demográficos, de comorbilidad, de la técnica dialítica (tiempo, frecuencia, tipos de membranas y flujo sanguíneo) y de los tratamientos recibidos en los campos de la anemia y del metabolismo mineral y óseo. Los indicadores de cumplimiento se refieren a las siguientes áreas: adecuación de diálisis, anemia, metabolismo mineral y óseo, nutrición, enfermedades víricas, accesos vasculares, mortalidad, morbilidad (ingresos hospitalarios) y trasplante $^{(5)}$.

En el HGC hay un promedio de cien pacientes en terapia de sustitución renal en espera de un trasplante de riñón. De éstos, alrededor de 35 se encuentran en hemodiálisis y los restantes en programa de diálisis peritoneal crónica ambulatoria.

En Costa Rica, los pacientes que se encuentran en el programa de hemodiálisis son divididos en 2 grupos: unos reciben 2 sesiones por semana de alrededor de 3 horas cada una y otros, 3 sesiones por semana con la misma duración, esto es debido a la carencia de infraestructura y recurso humano. Según su evolución clínica, pueden ser trasladados de grupo, para adecuar las horas de terapia dialítica y mejorar la calidad de vida. En vista de que no en todos los pacientes se están cumpliendo las horas de diálisis semanales mínimas sugeridas por las guias internacionales $^{(4)}$, se sospecha que tampoco se cumple con los indicadores de calidad descritos por las guías.

El objetivo del presente trabajo es presentar los primeros resultados sobre indicadores de calidad obtenidos en la Unidad de Hemodiálisis del HGC, para poder a partir de ahí detectar fortalezas y debilidades y proponer un plan de acción, con el fin de mejorar estos indicadores en el futuro cercano.

\section{Materiales y Métodos}

Diseño del Estudio: descriptivo, poblacional, de los indicadores obtenidos en el HCG en el año 2012 y primer trimestre del 2013.

Pacientes: En el año 2012, un promedio de 28.6 pacientes por mes, recibió hemodiálisis crónica. En el primer trimestre de 2013 el mismo promedio fue de 25.3. El total de pacientes estudiados en los diferentes momentos del estudio y para el cálculo de los diferentes indicadores analizados ha sido variable, en función de la incorporación de pacientes nuevos y las bajas existentes en la unidad, tanto por trasplante como por mortalidad. (Tabla 1)

No hubo ningún criterio de exclusión: todos los pacientes que estuvieron en la unidad en el momento del análisis y cuyos datos estuvieran incluidos en el programa informático, se incorporaron al análisis de indicadores.

Indicadores analizados: fueron los conocidos como de cumplimiento. Se refieren a las áreas de adecuación de diálisis, anemia, metabolismo mineral y óseo, nutrición, enfermedades víricas y accesos vasculares.

Metodología: Para la recopilación de la información se utilizó una base de datos en Excel, que es actualizada de forma mensual para cada uno de los pacientes en terapia. En dicha base de 
datos se incluyó los distintos indicadores según la periodicidad establecida en nuestra unidad:

- Perfil mensual: adecuación de diálisis, hemoglobina, calcio, fósforo, albúmina, lípidos

- Perfil Bimensual: PTH, ferritina

- Perfil anual: accesos vasculares, infecciones asociadas a catéter.

Tabla 1. Número de pacientes en hemodiálisis según mes y año. Unidad de Hemodiálisis. HCG.

\begin{tabular}{|ccc|}
\hline Mes & $\mathbf{2 0 1 2}$ & $\mathbf{2 0 1 3}$ \\
\hline Enero & 28 & 25 \\
\hline Febrero & 26 & 23 \\
\hline Marzo & 33 & 28 \\
\hline Abril & 36 \\
\hline Mayo & 31 \\
\hline Junio & 31 \\
\hline Julio & 28 \\
\hline Agosto & 35 \\
\hline Setiembre & 32 \\
\hline Octubre & 37 \\
\hline Noviembre & 26 \\
\hline Diciembre & 24 \\
\hline Fuente: base de datos del estudio & \\
\hline
\end{tabular}

Los resultados obtenidos fueron comparados con los índices recomendados por la Sociedad Española de Nefrología ${ }^{(5)}$, para su posterior análisis.

\section{RESULTADOS}

Los resultados obtenidos en los 15 meses analizados en este estudio han sido divididos en índices de calidad prioritarios y no prioritarios y los mismos se muestran en las tablas 2 y 3 respectivamente. Con respecto aquellos indicadores de los cuales no se cuenta con un registro adecuado de la información se hará referencia en la discusión de la situación en la que se encuentran en nuestra Unidad de Hemodiálisis

\section{DISCUSIÓN}

En este trabajo se presentan los resultados de la información obtenida en los últimos quince meses en la Unidad de Hemodiálisis del Hospital Calderón Guardia, los cuales han sido divididos en los que se obtuvieron el año 2012 y los del primer trimestre del 2013 para su eventual comparación. El análisis de estos resultados nos permite vislumbrar las futuras áreas de mejora y las potencialidades de esta estrategia de evaluación comparativa de los indicadores de calidad.

Tabla 2. Indicadores de Calidad Prioritarios en Hemodiálisis HCG

\begin{tabular}{|lccc|}
\hline \multicolumn{1}{|c}{ Indicador } & Recomendado & $\mathbf{2 0 1 2}$ & $\mathbf{2 0 1 3}$ \\
\hline Dosificación Diálisis $\mathrm{Kt} / \mathrm{V} \geq 1.2$ & $80 \%$ & $50.3 \%$ & $47.08 \%$ \\
\hline $\begin{array}{l}\text { Fistula arterio venosa desarrollada previo } \\
\text { ingreso HD }\end{array}$ & $\geq 80 \%$ & $<10 \%$ & $<10 \%$ \\
\hline Pacientes Según acceso vascular & $\begin{array}{c}\text { FAV autóloga: } \geq 80 \% \\
\text { Prótesis: }<10 \% \\
\text { Catéter: }<10 \%\end{array}$ & $\begin{array}{c}25 \% \\
5 \%\end{array}$ & $\begin{array}{c}12.5 \% \\
0 \%\end{array}$ \\
\hline Albúmina Sérica $<3.5 \mathrm{~g} / \mathrm{dl}$ & $<20 \%$ & $70 \%$ & $87.5 \%$ \\
\hline Fósforo $<5.5 \mathrm{mg} / \mathrm{dl}$ & $>75 \%$ & $58.2 \%$ & $71.5 \%$ \\
\hline $\begin{array}{l}\text { Hórmona Paratiroidea } \\
150-300 \mathrm{pg} / \mathrm{ml}\end{array}$ & $>30 \%$ & $36.5 \%$ & $49.5 \%$ \\
\hline $\begin{array}{l}\text { Hemoglobina } \\
\text { entre } 11-13 \mathrm{~g} / \mathrm{dl}\end{array}$ & $>95 \%$ & $18.6 \%$ & $26.5 \%$ \\
\hline Ferritina $100-800 \mu \mathrm{g} / \mathrm{dl}$ & $>80 \%$ & $18.4 \%$ & $14.6 \%$ \\
\hline Vacunación VHB & $100 \%$ & $58.1 \%$ & $65.3 \%$ \\
\hline Determinación AC VHC & $100 \%$ & $100 \%$ & $100 \%$ \\
\hline Incidencia Infección VHC & $0 \%$ & $100 \%$ & $100 \%$ \\
\hline
\end{tabular}

HD: hemodiálisis. AC: anticuerpos. VHC: Virus hepatitis C

Fuente: base de datos del estudio. 
Tabla 3. Indicadores de calidad No Prioritarios en Hemodiálisis HCG

\begin{tabular}{|lccc|}
\hline \multicolumn{1}{|c}{ Indicador } & Recomendado $^{(\mathbf{5})}$ & $\mathbf{2 0 1 2}$ & $\mathbf{2 0 1 3}$ \\
\hline Sepsis de Catéter Tunelizado & $<50 \%$ anual & $9.6 \%$ & \\
\hline LDL $<100 \mathrm{mg} / \mathrm{dl}$ & $>60 \%$ & $68.8 \%$ & $70.4 \%$ \\
\hline $\begin{array}{l}\text { Producto Calcio*fósforo } \\
<55 \mathrm{mg}^{2} / \mathrm{dl}^{2}\end{array}$ & $>65 \%$ & $43.9 \%$ & $55.3 \%$ \\
\hline $\begin{array}{l}\text { Hiperparatiroidismo Severo } \\
(\mathrm{PTH}>800 \mathrm{pg} / \mathrm{dl})\end{array}$ & $<0,05 \%$ & $14.6 \%$ & $12 \%$ \\
\hline Hemoglobina Media (g/dl) & $>11$ & 8.6 & 8.5 \\
\hline Tasa Vacunación antigripal & $100 \%$ & $100 \%$ & $100 \%$ \\
\hline Determinación VHC por PCR & $100 \%$ & $0 \%$ & $0 \%$ \\
\hline
\end{tabular}

LDL: lipoproteína de baja densidad. PTH: hormona paratiroidea. PCR: Reacción en cadena de la Polimerasa. Fuente: base de datos del estudio

Al analizar los indicadores de calidad prioritarios de hemodiálisis, de los cuales tenemos registro, se encontró que los únicos indicadores en los cuales se alcanzó las metas, son los que tienen que ver con infecciones víricas. Ésta es la consecuencia directa de que el $100 \%$ de los pacientes en programa de hemodiálisis en el $\mathrm{HCG}$ son referidos a la consulta de Infectología, en la cual se tamizan, desparasitan y vacunan de forma rutinaria y protocolizada. No se cuenta con PCR para la determinación de virus hepatitis $\mathrm{C}$, por lo que no es posible alcamar ese indicador.

En cuanto a la dosificación de diálisis, utilizando la ecuación de Daugirdas ${ }^{(6)}$, únicamente alrededor del 50\% tienen KT/V > 1.2 (Figura 1). Lo que indica que la mitad de los pacientes reciben terapia dialítica subóptima y un porcentaje de los pacientes con $\mathrm{KT} / \mathrm{V}>1.2$ probablemente tengan este indicador en rango apropiado, pero debido a un estado nutricional comprometido y dado que no es posible medir la tasa de producción de urea, se desconoce el porcentaje real de este grupo y debe ser inferido al observar los índices de hipoalbuminemia e hipocolesterolemia que tienen algunos pacientes. Para la mejoría en este indicador, se está trabajando en que en la unidad se introduzcan nuevos filtros, que permitan mejorar el aclaramiento, debido a que no se cuenta con la opción de aumentar el tiempo de diálisis por las limitaciones en relación a infraestructura y recurso humano

Con respecto a los indicadores relacionados con el acceso vascular, menos del $10 \%$ de los pacientes que ingresan al programa cuentan con un acceso venoso ya desarrollado, es decir, una fístula arteriovenosa lista para ser utilizada. Esto se debe a la captación tardía de los pacientes que requieren terapia de sustitución renal en los niveles de atención primaria, lo que hace que sea frecuente que en la primera valoración nefrológica sea en la que se indica tratamiento dialítico agudo de urgencia. A estos pacientes se les coloca un catéter de hemodiálisis, que puede ser temporal o permanente (según la disponibilidad del momento en el servicio) y muchas veces la programación para la cirugía para la realización de la fistula arteriovenosa es diferida.

En los casos en los que el paciente tiene un donador para un eventual trasplante, se toma la conducta de mantener como acceso el catéter, en vista de que el tiempo que tarda en la programación de la cirugía no sobrepasa en promedio los 4 a 6 meses. Por otro lado, las comorbilidades que en la mayoría de los casos tienen los pacientes que requieren de terapia de sustitución renal hacen que en muchas ocasiones no sea posible la realización de fístulas por enfermedad vascular periférica. A pesar de que la mayoría de los pacientes en hemodiálisis tienen catéter como acceso venoso, la incidencia de sepsis asociada a catéter del año pasado fue menor del $10 \%$, alcanzando así las metas recomendadas. No se tienen datos de la tasa de trombosis de fístulas.

Los indicadores de riesgo cardiovascular prioritarios incluyen niveles de albúmina, fósforo y PTH. Los niveles de albúmina en los pacientes de la unidad se encuentran persistentemente bajos y ésto habla de un estado crónico de inflamación que no se está controlando de forma adecuada con la hemodiálisis, asociado a un factor de deterioro nutricional asociado a la terapia. Es además un factor conocido que se asocia a la mortalidad en hemodiálisis ${ }^{(7)}$. 


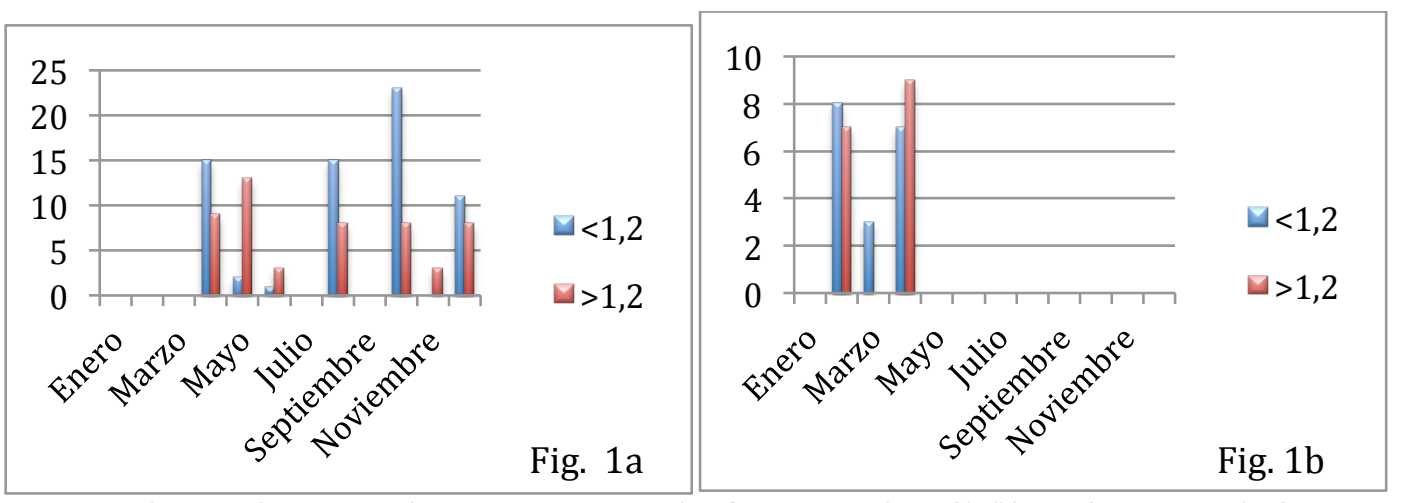

Figura 1. Distribución de número de pacientes según dosificación Diálisis: KT/V. 1a: Año 2012. 1 b: Año 2013. Fuente: Base de datos del estudio

En cuanto a los niveles de fósforo y PTH, si bien en ninguno de los dos indicadores se ha logrado alcanzar las metas, sí se ve una tendencia a la mejoría, principalmente en los niveles de $\mathrm{PTH}$, en relación con que se ha establecido de forma rutinaria y secuencial la toma de la PTH, permitiendo un seguimiento, detección $\mathrm{y}$ selección de pacientes para el uso del análogo selectivo de receptor de vitamina D (Paricalcitol). Sin embargo, la prevalencia de hiperparatiroidismo severo sigue siendo muy alta con respecto a lo recomendado. Se esperaría que en controles futuros este parámetro tenga mejoría con la continuación del uso del paricalcitol. En lo que se refiere al indicador que mide niveles de fósforo y el producto calcio*fósforo (Figura 2), evidentemente no se cumple con las metas recomendadas. Ésto se explica por el tiempo acortado de diálisis y la utilización de un quelante de fósforo (hidróxido de aluminio), que es mal tolerado por los pacientes y que además su recomendación es no extender su uso a más de ocho semanas $^{(4)}$.
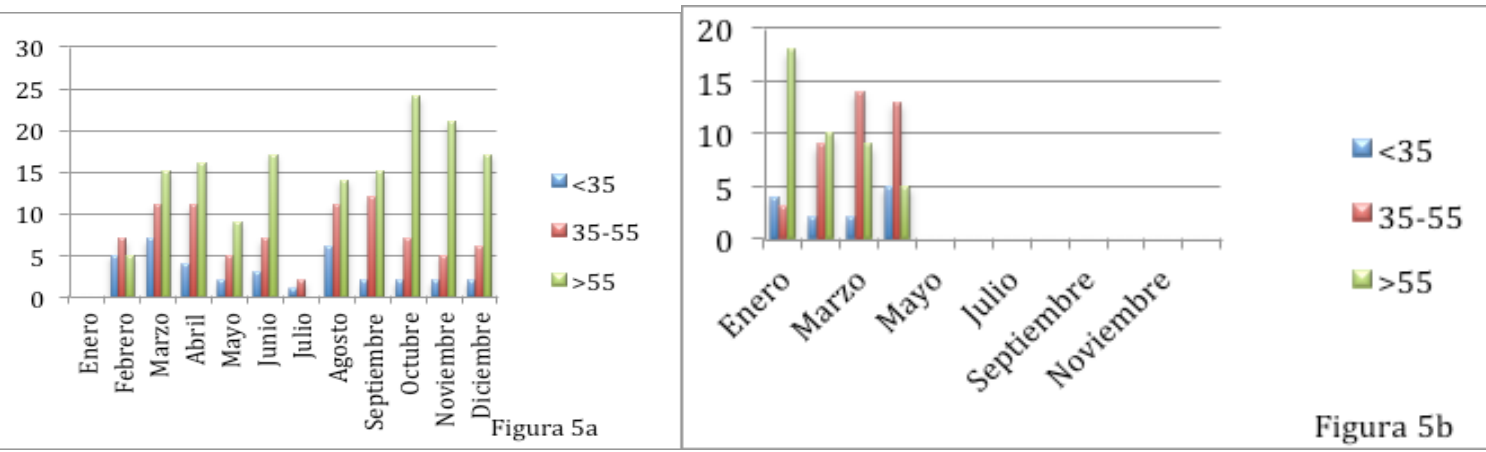

Figura 2. Distribución de número de pacientes según producto calcio*fósforo $\left(\mathrm{mg}^{2} / \mathrm{dl}^{2}\right)$. 5a: 2012, 5b:2013

Otro de los indicadores de riesgo cardiovascular, no prioritario es el nivel de colesterol LDL. En este punto la unidad alcanza y supera la meta establecida en las guías, sin embargo queda la duda de cuánto de esto en realidad está reflejando un estado de desnutrición severa, apoyado también por los bajos niveles de albúmina mencionados anteriormente. Sin embargo, no se cuenta con una forma objetiva de cuantificar el estado de nutrición de nuestros pacientes, ya que no se cuenta con personal de nutrición que de forma exclusiva de seguimiento a las necesidades de los mismos.

Uno de los indicadores de calidad más importantes y que sigue siendo más difícil de controlar es la anemia. Tanto así que se ha planteado la posibilidad de bajar meta de hemoglobina, que actualmente es que más de $95 \%$ de población se encuentre con niveles entre 11 y $13 \mathrm{~g} / \mathrm{dl}$, en vista de que son muy pocas las unidades de hemodiálisis que han podido 
alcanzar esta meta ${ }^{(7)}$, proponiéndose niveles de 10 a $12 \mathrm{~g} / \mathrm{dl}$. Este estudio evidencia que la situación de los pacientes del HCG no es diferente a la descrita anteriormente. Durante el 2012, únicamente $18.4 \%$ mantuvo cifras de $\mathrm{Hb}$ meta, mientras que en el 2013 la situación empeoró, cayendo a menos del 15\%. La $\mathrm{Hb}$ media fue alrededor de $8.5 \mathrm{~g} / \mathrm{dl}$. El año anterior el hospital agotó los reactivos para la cuantificación de ferritina, por lo que su medición fue inconstante, por esta razón y en vista de que los niveles bajos rondaban alrededor del $50 \%$ en las muestras tomadas, se decidió aplicar de forma rutinaria a todos los pacientes hierro intravenoso para asegurar así que los depósitos fueran restablecidos en su totalidad, además se les suplementa con sulfato ferroso y ácido fólico de forma crónica, para reponer pérdidas adicionales que son consecuencia de la hemodiálisis. La dosis de eritropoyetina semanal aplicada a los pacientes ronda en promedio de $170 \mu \mathrm{g} / \mathrm{kg} / \mathrm{semana}$ indicando resistencia a la eritropoyetina que se puede explicar por la inflamación, déficit de hierro y el uso de un quelante de fósforo con aluminio que ha demostrado alcanzar niveles de aluminio tóxico en otros hospitales de nuestro país.

Desafortunadamente, estos niveles de $\mathrm{Hb}$ obligan a someter a los pacientes a múltiples transfusiones, que pueden desencadenar un estado de hipersensibilización y complicar un trasplante renal futuro.

La calidad del agua que se utiliza en la unidad no es sometida a estudios de rutina por lo que no se cuenta con los niveles de endotoxinas o aluminio, ni con la conductividad, ni tampoco se hacen cultivos de la misma de forma rutinaria. Con respecto a este punto, solo se cuenta con los cultivos que de forma ocasional y no programada hace el comité de infecciones nosocomiales, en los cuales nunca se ha detectado crecimiento significativo de ningún germen. En el año 2012 se hizo el análisis de endotoxinas en líquido de diálisis como parte de una tesis de grado de Biología, Universidad de Costa Rica, que dio como resultado la presencia de endotoxinas en el agua de ingreso a las máquinas de diálisis y en el agua posterior a la purificación que hacen las máquinas. La exposición de la sangre de los pacientes al agua de diálisis con endotoxinas definitivamente desencadena inflamación $\mathrm{y}$ deteriora los indicadores anteriormente expuestos, parte de este proceso se ve evidenciado en los niveles persistentemente elevados de la proteína $\mathrm{C}$ reactiva (PCR).

Dentro de los parámetros que se consideran no prioritarios está también evaluar al menos una vez al año, el estado de salud mental y física percibidas por los pacientes en el programa de hemodiálisis crónica. Se realizó un análisis descriptivo estratificado transversal durante Diciembre del 2012, con la idea de documentar al prevalencia de depresión y ansiedad de los usuarios mayores de 18 años del servicio de hemodiálisis del HCG durante diciembre del 2012 (Tesis Dra. Andrea Medina, en espera de publicación). Para este fin se aplicó el inventario de depresión de Beck y el inventario de ansiedad de Beck. Con respecto al inventario de depresión, se detectó que ésta se encontraba en el $57 \%$ de los casos y predominando la de leve intensidad $22 \%$ seguido de la severa $13 \%$. En relación con la ansiedad predominó la normal (43\%) seguido de la severa con un $26 \%$. Luego de que se conocieran estos datos, se puso en práctica varias estrategias para mejorar la calidad de vida de los pacientes, que incluyen el apoyo constante de una enfermera de salud mental, la cual además de brindarles apoyo continuo individualizado les imparte talleres de relajación. Una vez mantenido este plan de trabajo durante 6 meses se analizará su efectividad para disminuir el grado de ansiedad y depresión realizando de nuevo los mismos cuestionarios

\section{CONCLUSIONES Y RECOMENDACIONES}

Ya ha sido demostrada la asociación entre el grado de cumplimiento de los estándares de los sistemas de monitorización y la morbimortalidad de los pacientes en hemodiálisis crónica, por lo que constituyen una de las herramientas más útiles para mejorar los resultados clínicos $^{(2)}$. La monitorización de los indicadores permite valorar si los criterios de calidad establecidos se cumplen o no y si la prestación brindada a los pacientes cumple con ellos.

Al evaluar los resultados de los indicadores y las expectativas en cada uno de ellos, hay que tener en cuenta que no se aplicó ningún criterio de exclusión. Al análisis de indicadores fueron incorporados todos los pacientes, sin importar el tiempo desde su incorporación en la unidad. Sin embargo, el panorama global indica que unidad de hemodiálisis del HCG no está cumpliendo con 
las metas propuestas por las guías internacionales, y ante de la falta de datos de las otras unidades de hemodiálisis en Costa Rica, estos resultados pueden resultar orientativos a la situación global. Dichos resultados demuestran la importancia de definir estándares que se ajusten de manera adecuada a las características de la población que se está tratando, a los nueas estrategis terapéuticas disponibles $\mathrm{y}$ de revisarlos $\mathrm{y}$ cambiarlos, de forma periódica si es preciso.

Uno de los objetivos principales de este estudio fue proponer un plan de acción para logar mejorar los indicadores y así prolongar la sobrevida y mejorar la calidad de vida de los pacientes. Se debe reforzar la educación en el primer y segundo nivel de atención para una detección temprana de la enfermedad renal crónica y que el médico especialista en nefrología tenga un mayor plan de acción para preparar a los pacientes previo al ingreso al programa de sustitución renal. Se necesita mejorar el KT/V utilizando la medida mejor costo efectiva para el paciente y para la institución, por lo que se propone que el primer cambio sea aumentar el tamaño de los dializadores.

En cuanto al manejo del metabolismo mineral y óseo, éste es el apartado donde se ha producido más cambios a nivel internacional, debido a la introducción en los últimos años de cambios conceptuales que han llevado el surgimiento de nuevas estrategias terapéuticas ${ }^{(8)}$. Es fundamental poder llevar estas nuevas alternativas de tratamiento a los pacientes de nuestro país, incluidos los nuevos quelantes de fósforo, para poder así alcanzar las metas en cuanto a niveles de fósforo y producto calcio*fósforo y evitar los niveles tóxicos de aluminio por su ingesta crónica.

Otro de los problemas detectados fue la mala calidad del agua utilizada en la unidad de diálisis, que fue demostrado por las altas cantidades de endotoxinas encontradas tanto antes como después del proceso de purificación que hace la máquina. Considerando que estas endotoxinas son parte de la justificación del estado de inflamación permanente y este a su vez perpetua la anemia, resulta prioritario que cualquier unidad de hemodiálisis de Costa Rica cuente con una planta de purificación de agua para diálisis.
El estado de nutrición de los pacientes de la unidad es en general malo y como se mencionó previamente, dentro del equipo humano que trabaja directamente con estos pacientes no se cuenta con una nutricionista que atienda esta necesidad ni tampoco con un equipo de bioimpedancia que permita objetivizar este parámetro, por esta razón se considera que las unidades de hemodiálisis deberían de contar con una nutricionista especializada que brinde la atención directa los pacientes en terapia renal.

Con respecto a los niveles de depresión y ansiedad detectados en la unidad ya se pusieron en práctica medidas para mejorar estos indicadores y esta pendiente la revaloración de los mismos luego de 6 meses de implementadas estas estrategias.

Una de las barreras que impide alcanzar las metas propuestas es la que tiene que ver con los factores socioeconómicos e incluye la no adherencia al tratamiento y el ausentismo a las citas programadas, para mejorar en este sentido se ha implementado un programa de educación en conjunto con Farmacia y trabajo social.

Este estudio demostró que se debe encontrar el equilibrio entre lo óptimo y lo alcanzable, pero para lograr esto se debe de tener una adecuada recolección de datos que permitan objetivizar la situación real de las unidades de hemodiálisis del país. No se puede obviar la sobrecarga de trabajo que supone la elaboración, implantación y análisis de un sistema de monitorización. Por ello, una limitación evidente es la necesidad de diseñar herramientas automáticas que faciliten su cálculo.

Se puede concluir que se está avanzando en lo referente al conocimiento de los resultados de la hemodiálisis, aunque el trabajo pendiente todavía es extenso, y que la monitorización de indicadores de calidad respecto a un estándar y su puesta en común con otros centros puede contribuir a la mejora de resultados y a la disminución en la variabilidad entre centros

\section{REFERENCIAS BIBLIOGRÁFICAS}

1. U.S. Renal Data System. USRDS 2003 Annual Data Report: Atlas of End-Stage Renal Disease in the United States. Bethesda, MD: National Institutes of Health, National 
Institute of Diabetes and Digestive and Kidney Diseases; 2003.

2. Rocco M Frankenfield D Hopson S McClellan W. Relationship between Clinical Performance Measures and Outcomes among Patients Receiving Long-Term Hemodialysis. Ann Intern Med. 2006;145:512519.

3. Collins AJ. Influence of target hemoglobin in dialysis patients on morbidity and mortality. Kidney Int Suppl. 2002;80:44-48. [PMID: 11982812]

4. http://www.kidney.org/professionals/k doq

5. http://www.senefro.org/

6. Daugirdas JT. Second generation logarithmic estimates of single-pool variable volume $\mathrm{Kt} / \mathrm{V}$ : an analysis of error. J Am Soc Nephrol 1993;4:1205-1213.

7. Alcázar JM. Estudio multicéntrico de calidad en hemodiálisis. Nefrología 2008;6: 597-606.

8. Arenas MD Álvarez-Ude F Gil MT et al. Implementation of K/DOQI Clinical Practice Guidelines for Bone Metabolism and Disease in Chronic Kidney Disease after the introduction of cinacalcet in a population of patients on chronic hemodialysis. Nephrol Dial Transplant 2007;22(6):16391644.

\section{CONFLICTO DE INTERÉS}

En el presente trabajo no existió ningún conflicto de interés. 